\title{
AUTOCONCEITO E DESEMPENHO ACADÊMICO EM ALUNOS DE PSICOLOGIA
}

\author{
Self-Concept And Academic Performance In \\ Psychology Students
}

Adriana Cristina Boulhoça Suehiro

\section{Resumo}

O autoconceito tem sido estudado nas diversas áreas da Psicologia em virtude da importância que assume na compreensão do processo do desenvolvimento humano. Tendo em vista a relevância do autoconceito na vida de um indivíduo, o presente trabalho objetivou verificar a relação existente entre o autoconceito e 0 desempenho acadêmico em estudantes do ensino superior. Fizeram parte desse estudo 142 estudantes do segundo e oitavo semestres do curso de Psicologia, com idade entre 17 e 58 anos, dos períodos diurno e noturno de uma Universidade particular do interior paulista. Os estudantes que assinaram o Termo de Consentimento Livre e Esclarecido preencheram, em horánio de aula previamente cedido pelo professor, um questionário de identificação dos sujeitos e, em seguida, a Escala Reduzida do Autoconceito - ERA. Os principais resultados obtidos evidenciaram a existência de indicadores de correlação entre o autoconceito e o desempenho acadêmico, todavia esses resultados não nos permitem concluir definitivamente quanto à existência dessa correlação. Talvez esses resultados sejam decorrentes de um tipo de avaliação que não discrimina os alunos ou os diferencia muito pouco.

Palavras-chave: autoconceito, desempenho acadêmico, ensino superior.

1 Psicóloga. Doutoranda em Psicologia pelo Programa de Pós-Gradução Stricto Sensu em Psicologia da Universidade São Francisco/Itatiba. Bolsista CAPES. Endereço para correspondência: Rua José Strazzacapa, 91- Campinas/SP - CEP 13091-130 - e-mail: dricbs@uol.com.br 


\section{Abstract}

The self-concept has been studied in the diverse areas of psychology in virtue of the importance that assumes in the understanding of the process of the human development. In view of the relevance of the self-concept in the life of an individual, the present work objectified to verify the existing relation between the self-concept and the academic performance in students of superior education. 142 students of second and the eighth semesters of the course of Psychology had been part of this study, with age between 17 and 58 years, of the daylights and nocturnal of a particular university of the São Paulo interior. The students who had signed the term of free and clarified assent had filled, in schedule of class previously yielded by the professor, a questionnaire of identification of the citizens and, after that, reduced scale of the self-concept - ERA. The main gotten results had evidenced the existence of pointers of correlation between the self-concept and the academic performance, however these results in do not allow them to conclude definitively how much to the existence of this correlation. Perhaps these results are decurrent of a type of evaluation that does not discriminate the pupils or it differentiates them very little.

Keywords: autoconceito, academic performance, superior education.

O auto conceito tem sido estudado nas diversas áreas da Psicologia em virtude da importância que assume na compreensão do processo do desenvolvimento humano (Cortesão \& Torres, 1984; Vaz Serra, 1986; Vaz Serra \& Firmino, 1986; Veiga, 1988,1989; entre outros). Sua investigação teórica é caracterizada por uma grande imprecisão da terminologia e discordâncias no que se refere às definições empregadas. Todavia, embora a literatura não revele uma definição operacional clara, concisa e universalmente aceita, há uma certa concordância entre os vários autores em relação à definição geral do termo. Dentro desse contexto, o autoconceito é definido, basicamente, como a percepção que o indivíduo tem de si (Jacob \& Loureiro, 1999; Loureiro, 2001; Marsh, 1984; Marsh, Byrne \& Shavelson, 1988; Sossai, 1975; Stevanato \& Loureiro, 2000; Vaz Serra, 1988).

Admitindo as deficiências terminológicas e a grande multiplicidade de conceitos encontrados na literatura, trabalhar-se-á nesse estudo com a definição operacional adotada por Albuquerque e Oliveira (2002), na qual afirmam que o autoconceito é composto por imagens acerca de como o indivíduo pensa que é, do que pensa que os outros pensam de si, do que pensa ser capaz de realizar e de como gostaria de ser. A definição apresentada pelos autores remete o leitor à multidimensionalidade do constructo e evidencia a necessidade de uma maior caracterização para que sua compreensão se torne mais viável e menos densa. Logo, o autoconceito é formado por um conjunto de atribuições cognitivas que um indivíduo faz de si, de seu comportamento em diferentes situações objetivas e das suas características pessoais. Ele envolve três tipos principais de percepção, a saber: descritiva (avaliações conscientes de si no que diz respeito a capacidades e competências); avaliativa (respostas emocionais positivas ou negativas) e de congruência (discrepância entre a auto-avaliação e a avaliação externa) (Candieux, 1996).

Tanto o autoconceito quanto seus constituintes são aprendidos, pois é no relacionamento com as outras pessoas que os indivíduos desenvolvem suas percepções e constroem suas crenças a respeito de si mesmos, de suas capacidades e adquirem a capacidade de valorização de seus potenciais. A interação com as demais pessoas é responsável por muitos outros aprendizados do indivíduo extremamente relevantes na constituição do autoconceito, dentre os quais se destacam as expectativas quanto às suas capacidades de êxito nas ações que empreende pela sua experiência e pela observação das ações das pessoas que o cercam, bem como pelas conseqüências destas.

Sabe-se que há uma multiplicidade de fatores que influenciam a aprendizagem escolar e 0 desempenho acadêmico e que os fatores que se relacionam com o processo do aprender envolvem tanto questões pessoais e internas do estudante, quanto questões relacionadas à família e ao grupo social no qual está inserido, bem como questões mais amplas relativas à escola, enquanto contexto de aprendizagem influenciado pelas interações, medidas pedagógicas e decretos governamentais que regem o ensino. Tais fatores se inter-relacionam e se determinam mutuamente influenciando o desempenho acadêmico de um indivíduo.

Dentre os fatores relacionados com o próprio indivíduo e que influenciam o processo de aprendizagem escolar, deve-se considerar as questões relacionadas a fatores orgânicos, cognitivos, afetivos e motivacionais. Alterações orgânicas e 
neurológicas podem influenciar negativamente as capacidades cognitivas e adaptativas do estudante, gerando rebaixamento intelectual e prejudicando capacidades necessárias para um bom desempenho escolar. Contudo, de acordo com Jacob (2001), muitos estudantes sem alterações orgânicas ou cognitivas apresentam dificuldades de aprendizagem, o que poderia ser explicado pela interferência de fatores afetivos e das funções adaptativas na aprendizagem.

Um outro fator associado ao desempenho acadêmico dos estudantes diz respeito ao papel fundamental do professor em todo o processo. A conduta do professor, enquanto agente avaliador, em relação ao aluno será determinante para o seu autoconceito e resultados acadêmicos positivos, bem como o conceito que o professor faz de si mesmo (Cubero \& Moreno, 1995). Assim, verifica-se a necessidade da utilização de formas eficazes de avaliação da aprendizagem, nas quais o professor estabeleça não só objetivos pedagógicos, mas objetivos realistas e condizentes com as possibilidades de seus alunos, buscando se aproximar ao máximo de uma prática avaliativa mais construtiva como têm preconizado inúmeras pesquisas (Almeida, 1997; Luckesi, 2001, Pinto, 1996; Serrano, 1991; Sordi, 2000; Sousa, Depresbiteris, Franco \& Sousa, 1995; Sousa, 1995).

Dentro dessa perspectiva, Bzuneck (2001) tem ressaltado a importância de que se avalie o processo de aprendizagem como um todo e de que o professor seja concebido como aquele que deve conduzir seus alunos a perceberem seus erros e fracassos como uma possibilidade no processo de construção do conhecimento e não como resultante da falta de capacidade dos mesmos. Diante dessa realidade, a aplicação de provas desempenha outras funções, além das tradicionais, e a avaliação da aprendizagem passa a incluir a mediação do pensamento sintético, das aquisições e aplicações da aprendizagem, bem como a metacognição e as estratégias de aprendizagem dos estudantes, o que, de acordo com Sisto (2001), contribuiria para que o professor planeje o ensino de modo a potencializar a aquisição do conhecimento por parte de seus alunos. Todos esses aspectos citados em relação à conduta do professor requerem, segundo Serrano (1991), que ele esteja consciente da importância da situação de aprendizagem em sala de aula, das avaliações e feedbacks na formação do auto- conceito do estudante, colocando-se assim como elemento ativo nessa formação.

As relações entre o autoconceito e o desempenho acadêmico têm sido estudadas por diversos autores brasileiros e estrangeiros com crianças e alunos do ensino médio. Tais pesquisas apontam que os conhecimentos e sentimentos positivos em relação a si próprio repercutem no bom funcionamento individual, na motivação e na forma como o estudante responde às demandas da aprendizagem, destacando-se a presença de correlações positivas entre estes dois constructos (Abu-Hilal, 2000; Amorim, 2000; Brookver, Thomas \& Patterson, 1964; Castro, 1999; Estêvão \& Almeida, 1999; Hamachek, 1995; Hosley, Hooper \& Gruber, 1998; Jacob, 2001; Sink, Barnett \& Pool, 1993; Souza, 1996).

Rosemberg et al. (1989, citado por Castro, 1999), por exemplo, em estudo com estudantes da $10^{\underline{a}}$ e $11^{a}$ séries do sistema escolar americano observou que as notas (avaliações) têm um impacto maior sobre a auto-estima do que sobre 0 desempenho. Em uma nova análise dos dados referentes a essas mesmas séries, Marsh, citado pela mesma autora, demonstrou a importância das notas sobre o autoconceito geral e acadêmico dos estudantes, chegando mais tarde à conclusão de que 0 autoconceito tem forte efeito sobre o desempenho acadêmico. Também Shavelson e Bolus (1982) encontraram evidências da predominância causal do autoconceito sobre o desempenho acadêmico em sua pesquisa, realizada em dois momentos separados por um período de seis meses de intervalo, com estudantes da 7a e 8a séries do Ensino Médio, antigo primeiro grau.

A influência de outros fatores tanto sobre o desempenho quanto o autoconceito vem sendo estudada por muitos estudiosos da área. No que se refere à idade, estudos evidenciam que 0 autoconceito se modifica com o passar dos anos, porém há a necessidade de estudos longitudinais que comprovem essa mudança (Engel, 1959; Wylie, 1968, citado por Sossai, 1975). Outras pesquisas buscam analisar a relação entre o autoconceito, desempenho acadêmico e a variável gênero. Em relação a essa variável, os resultados são bastante divergentes. Alguns estudos concluem que não há diferenças significativas entre 0 autoconceito de homens e mulheres, embora os primeiros apresentem um rendimento acadêmico menor (Hugues, 1968; Offer, 1971; Wylie, 1961; Castro, 1999). 
Tendo em vista os aspectos considerados, o presente estudo teve por objetivos: (a) identificar e comparar o autoconceito dos universitários do $2^{\underline{o}}$ e $8^{0}$ semestres do curso de Psicologia, com base na escala utilizada, (b) verificar a relação existente entre o Autoconceito e o Desempenho Acadêmico dos alunos e (c) estabelecer eventuais diferenças entre estas duas variáveis considerando a idade, o turno e o gênero dos sujeitos analisados.

\section{Método}

\section{Participantes}

Participaram deste estudo 142 alunos, sendo 73 que estão cursando o segundo semestre e 69 que estão cursando o oitavo semestre do curso de psicologia, de uma universidade particular do interior paulista. Os estudantes apresentavam média de idade de 24 anos, variando de 17 a 58 anos, com desvio-padrão de 6,58. A maioria pertencia ao gênero feminino $(86,6 \%)$ e ao período noturno $(66,2 \%)$.

\section{Material}

Foram utilizados: (a) um questionário de identificação dos sujeitos que versava sobre os seguintes aspectos: nome, registro acadêmico, idade, gênero e turno, elaborado pela autora (b) a Escala Reduzida do Autoconceito - ERA de Corona (2001), composta por 30 frases, sendo que em cada uma os sujeitos poderiam assinalar com um X o grau (Discordo totalmente, Discordo parcialmente, Indeciso, Concordo parcialmente ou Concordo totalmente) que melhor o descreveria. A escala tem o objetivo de avaliar o autoconceito, a auto-imagem e a autopercepção individual, partindo de três categorias do Eu moral e o Eu social. O autoconceito é classificado em (a) desvalorizado (abaixo de 109,16 pon- tos), (b) mal estruturado (entre 109,16 e 121,58 pontos), (c) bem estruturado (entre 121,59 e 134 pontos) ou (d) supervalorizado (acima de 134 pontos) de acordo com o escore alcançado pelo indivíduo. Faz-se importante ressaltar que embora a autora do instrumento utilize como critério de classificação do autoconceito os escores obtidos, não há especificação do que cada uma dessas classificações signifique. Para as análises foram consideradas: (a) as notas escolares, que foram resultantes das médias em avaliações acadêmicas do primeiro semestre do ano letivo, obtidas na Secretaria da Instituição, de cada uma das disciplinas de conteúdos específicos desse período do curso e (b) o escore obtido pelos universitários na Escala Reduzida do Autoconceito.

\section{Procedimento}

As provas foram aplicadas em uma sessão, em horário de aula previamente cedido pelo professor, aos alunos ingressantes e concluintes do curso que assinaram o Termo de Consentimento Livre e Esclarecido. Inicialmente os alunos preencheram, em sala de aula, o questionário de identificação do sujeito e, em seguida, a Escala Reduzida de Autoconceito.

\section{Resultados}

Os resultados foram analisados qualitativa e quantitativamente e, tendo em vista os objetivos pretendidos, utilizaram-se provas de estatística descritiva, a correlação de Pearson, o t de Student e a análise de variância no tratamento dos dados obtidos. Os dados referentes ao objetivo de identificar e comparar o autoconceito dos universitários do segundo e oitavo semestres do curso de Psicologia, com base na escala utilizada, encontram-se na Tabela 1. 
Tabela 1 - Comparação entre o número de respostas dadas pelos estudantes nas categorias de a utoconceito $(\mathrm{N}=142)$.

\begin{tabular}{|c|c|c|c|c|}
\hline Categorias Propostas & \multicolumn{2}{|c|}{ Segundo Semestre } & \multicolumn{2}{|c|}{ Oitavo Semestre } \\
\hline Autoconceito Desvalorizado & 13 & 17,8 & 11 & 15,94 \\
\hline Autoconceito Mal Estruturado & 20 & 27,40 & 15 & 21,74 \\
\hline Autoconceito Bem Estruturado & 31 & 42,47 & 32 & 46,38 \\
\hline Autoconceito Supervalorizado & 09 & 12,33 & 11 & 15,94 \\
\hline Total de alunos & 73 & - & 69 & - \\
\hline
\end{tabular}

Para verificar possíveis diferenças em função da pontuação na Escala Reduzida do Autoconceito, dividiram-se os sujeitos de acordo com os escores alcançados, obedecendo-se os critérios de classificação do autoconceito preconizados pelo instrumento utilizado (valores dos escores). Desse modo, obtiveram-se quatro grupos: Grupo 1 - estudantes que tiveram escores abaixo de 109,16 (autoconceito desvalorizado); Grupo 2 - estudantes que tiveram escores entre 109,16 e 121,58 (autoconceito mal estruturado); Grupo 3 - estudantes que tiveram escores entre 121,59 e 134 (autoconceito bem es- truturado) e Grupo 4 - estudantes que tiveram escores acima de 134 (autoconceito supervalorizado). A proporção de respostas quanto às categorias de autoconceito propostas pela escala diferiu pouco entre os semestres. O Autoconceito Desvalorizado foi apontado com maior freqüência pelos alunos do segundo semestre $(17,78 \%)$, enquanto que os universitários do oitavo semestre superaram os do segundo em termos de indicação da categoria Autoconceito Supervalorizado (11\%). Os escores obtidos pelos universitários na escala de autoconceito utilizada estão apresentados na Tabela 2.

\section{Tabela 2 - Pontuação obtida pelos universitários na escala de a utoconceito aplicada $(\mathrm{N}=142)$.}

\begin{tabular}{|l|l|l|l|l|}
\hline Autoconceito & Pontuação Média & Pontuação Mínima & Pontuação Máxima & Desvio Padrão \\
\hline Segundo Semestre & 120,89 & 84,00 & 140,00 & 12,61 \\
Oitavo Semestre & 122,29 & 74,48 & 147,00 & 13,82 \\
\hline Total de alunos & $\mathbf{1 2 1 , 5 7}$ & $\mathbf{7 4 , 4 8}$ & $\mathbf{1 4 7 , 0 0}$ & $\mathbf{1 3 , 1 8}$ \\
\hline
\end{tabular}

O desempenho na Escala Reduzida do Auto conceito - ERA apresentou índices de correlação marginalmente significativa entre os resultados obtidos e as médias $(\mathrm{r}=0,160$ e $\mathrm{p}=$ 0,057), quando considerada a totalidade dos alunos pesquisados. A pontuação máxima alcan- çada pelos estudantes universitários foi, respectivamente, de 147 pontos para o oitavo semestre e 140 para o segundo. A Tabela 3 apresenta as médias semestrais obtidas pelos estudantes de psicologia durante o primeiro semestre do ano letivo. 
Tabela 3 - Médias semestrais em avaliações acadêmicas obtidas pelos estudantes no curso de Psicologia $(\mathrm{N}=142)$.

\begin{tabular}{|l|l|l|l|l|}
\hline Médias & Pontuação Média & Pontuação Mínima & Pontuação Máxima & Desvio Padrão \\
\hline $\begin{array}{l}\text { Segundo Semestre } \\
\text { Oitavo Semestre }\end{array}$ & 7,48 & 5,40 & 8,97 & 0,71 \\
\hline Média Total & $\mathbf{7 , 6 5}$ & 5,38 & 9,97 & 0,93 \\
\hline
\end{tabular}

Como pode ser observado, os alunos do oitavo semestre apresentaram um melhor desempenho acadêmico, tendo uma pontuação média maior e também uma pontuação máxima mais elevada que os do segundo semestre. Com base na análise de variância, verificou-se que existe uma diferença marginalmente significativa $(\mathrm{F}(3,69)=2,399 ; \mathrm{p}=0,075)$ entre as médias semestrais dos estudantes do segundo semestre do cur- so quando comparados em função do autoconceito apresentado. O teste post-hoc de Tukey apontou que a diferença verificada está entre os grupos 1 (autoconceito desvalorizado) e 4 (autoconceito supervalorizado) ( $\mathrm{p}=0,005)$. As Tabelas 4 e 5 apresentam os dados referentes ao objetivo de estabelecer correlações entre 0 autoconceito e 0 desempenho acadêmico considerando a idade, o turno e o gênero dos sujeitos analisados.

\section{Tabela 4 - Comparação entre o número de respostas nas categorias de auto- conceito, considerando-se o gênero $(\mathrm{N}=142)$.}

\begin{tabular}{|c|c|c|c|}
\hline Categorias Propostas & $\begin{array}{l}\text { Masculino } \\
\mathbf{N} \quad \%\end{array}$ & $\begin{array}{l}\text { Fen } \\
\mathbf{N}\end{array}$ & $\underset{\%}{\operatorname{ino}}$ \\
\hline Autoconceito Desvalorizado & 15,79 & 21 & 17,08 \\
\hline Autoconceito Mal Estruturado & 21,05 & 31 & 25,20 \\
\hline Autoconceito Bem Estruturado & 52,63 & 53 & 43,09 \\
\hline Autoconceito Supervalorizado & 10,53 & 18 & 14,63 \\
\hline Total de alunos & 19 & 123 & - \\
\hline
\end{tabular}

Embora o gênero feminino (N=123) prevaleça sobre o masculino $(\mathrm{N}=19)$ não houve diferença significativa entre as mulheres e os homens nos escores do autoconceito $(t=0,113 ; p=0,910)$. Tanto os universitários do gênero masculino quanto os do feminino apontaram, respectivamente, com maior freqüência a categoria Autoconceito Bem Estruturado $(\mathrm{N}=10$ e $\mathrm{N}=53)$ e com menor freqüência a categoria Autoconceito Supervalonizado ( $\mathrm{N}=2$ e $\mathrm{N}=18$ ).

Comparando-se a distribuição dos universitários do gênero masculino e feminino quan- to ao desempenho acadêmico e ao autoconceito apresentado, observou-se que não houve diferenças significativas em ambos os grupos, constatadas pela análise de variância. Para os homens, obteve-se $(F(3,15)=1,72 ; \mathrm{p}=0,914)$ e para as mulheres $(F(3,119)=1,919 ; p=0,130)$. No que se refere ao turno, a análise de variância revelou a existência de diferença significativa $(F(3,44)=4,356 ; p=0,009)$ entre as médias semestrais e o autoconceito identificado dos universitários do período diurno. 
Para verificar possíveis diferenças em função da pontuação na Escala Reduzida do Autoconceito, dividiram-se os sujeitos de acordo com as faixas etárias. Desse modo, obtiveram-se três grupos: Grupo 1 - estudantes com faixa etária de 17 a 24 anos; Grupo 2 - estudantes com faixa etária de 25 a 32 anos; Grupo 3 - estudantes com faixa etária acima de 32 anos. A comparação entre o número de respostas nas categorias de autoconceito e cada uma dessas faixas etárias encontra-se na Tabela 5.

\section{Tabela 5 - Comparação entre o número de respostas nas categorias de auto- conceito, considerando-se a faix a etária $(\mathrm{N}=141)$.}

\begin{tabular}{|l|ll|ll|lc|}
\hline \multicolumn{7}{|c|}{ Faixas Etárias } \\
\hline Categorias Propostas & \multicolumn{2}{|c|}{$\begin{array}{l}\text { De 17 a 24 anos } \\
\text { N }\end{array}$} & $\begin{array}{l}\text { De 25 a 32 anos } \\
\text { N }\end{array}$ & \multicolumn{2}{c|}{$\begin{array}{c}\text { Acima de 32 anos } \\
\text { N }\end{array}$} \\
\hline Autoconceito Desvalorizado & 20 & 20,6 & 02 & 07,4 & 02 & 11,8 \\
Autoconceito Mal Estruturado & 27 & 27,8 & 05 & 18,5 & 02 & 11,8 \\
Autoconceito Bem Estruturado & 40 & 41,2 & 13 & 48,1 & 10 & 58,8 \\
Autoconceito Supervalorizado & 10 & 10,3 & 07 & 25,9 & 03 & 17,6 \\
\hline Total de alunos & 97 & - & 27 & - & 17 & - \\
\hline
\end{tabular}

Como pode ser observado, o Autoconceito Bem Estruturado foi apontada com maior freqüência pelos alunos de todas as faixas etárias, enquanto que os universitários com faixa etária de 17 a 24 anos superaram os das demais faixas no que se refere à indicação da categoria Autoconceito Desvalorizado. Quando se considera a faixa etária, não há diferença significativa $(\mathrm{F}(2,138)=0,408$; $\mathrm{p}=0,666$ ) entre os grupos e os estudantes mais velhos que, embora apareçam em menor quantidade $(\mathrm{N}=17)$, são os que apresentam melhor desempenho acadêmico (média semestral=7,82).

\section{Discussão e Considerações Fina is}

Partindo-se do pressuposto de que o autoconceito tem um papel mediacional em todo 0 processo de aprendizagem, não se pode compreender a conduta escolar do estudante, nesse caso especificamente do universitário, sem se considerar as percepções e os sentimentos que eles apresentam em relação a si mesmos e à sua própria competência acadêmica.

Diante do exposto, o presente estudo objetivou identificar e comparar o autoconceito dos universitários do $2^{\underline{0}}$ e $8^{\circ}$ semestres do curso de Psicologia, com base na escala utilizada, bem como verificar a relação existente entre o Autoconceito e o Desempenho Acadêmico dos alunos e, por fim, estabelecer correlações entre estas duas variáveis, considerando a idade, o turno e o gênero dos sujeitos analisados.

No que se refere ao primeiro objetivo proposto, verificou-se que a proporção de respostas, quanto às categorias de autoconceito, diferiu pouco entre os semestres, sendo que o Autoconceito Desvalorizado foi apontado com maior freqüência pelos alunos do segundo semestre, enquanto que os universitários do oitavo semestre superaram os do segundo em termos de indicação da categoria autoconceito Supervalorizado. Os universitários do oitavo semestre apresentaram um melhor desempenho acadêmico em relação aos do segundo, tendo uma pontuação média maior e também uma pontuação máxima mais elevada. Os resultados obtidos foram parcialmente incongruentes aos de outras pesquisas, como a de Jesus e Gama (1991), cujas evidências demonstram que as expectativas de sucesso e autoconceito de competências diminuem à medida que o estudante progride nas séries escolares, enquanto que, de maneira geral, o inverso é observado em relação ao desempenho ou rendimento acadêmico.

Os resultados apresentados na correlação entre o Autoconceito e o Desempenho Acadêmi- 
co dos alunos mostraram que o desempenho na Escala Reduzida do Autoconceito apresentou índices de correlação marginalmente significativa entre os resultados obtidos e as médias, quando considerada a totalidade dos alunos pesquisados. Tal nível de significância se repete quando consideradas as médias semestrais dos estudantes do segundo semestre do curso, sendo que a diferença verificada está entre os grupos 1 (autoconceito desvalorizado) e 4 (autoconceito supervalorizado). Dessa forma, as análises realizadas expressaram indicadores de correlação entre as duas variáveis focalizadas, mas não possibilitam afirmar a existência de correlação entre elas, o que está em dissonância com os resultados obtidos por outros estudos brasileiros e estrangeiros que têm apontado para a relação positiva entre 0 autoconceito e 0 desempenho acadêmico de estudantes (Abu-Hilal, 2000; Amorim, 2000; Burns, 1979; Brookver, Thomas \& Patterson, 1964; Castro, 1999; Hamachek, 1995; Serrano, 1991).

Talvez os resultados obtidos nesse estudo sejam decorrentes de um tipo de avaliação que não discrimina os alunos ou os diferencia muito pouco. Dessa forma, como pontua Serrano (1991), o professor surge como um elemento de grande importância no processo de aprendizagem do estudante, pois, enquanto agente avaliador, possui grande influência sobre as avaliações que os alunos fazem sobre si e sobre o seu próprio desempenho. Nesse sentido, verifica-se a necessidade da utilização de formas eficazes de avaliação da aprendizagem dos estudantes do ensino superior, especialmente para que as falhas detectadas sejam superadas. Diante do papel fundamental dessa avaliação, inúmeras pesquisas têm sido realizadas com o intuito de torná-la mais prática e construtiva (Almeida, 1997; Luckesi, 2001; Sordi, 2000; Sousa, Depresbiteris, Franco \& Sousa, 1995; Sousa, 1995).

Bzuneck (2001), entre outros autores, tem criticado a concepção da avaliação como um produto final, ressaltando a importância de que se avalie o processo de aprendizagem como um todo e de que o professor seja concebido como aquele que deve conduzir seus alunos a perceberem seus erros e fracassos como uma possibilidade no processo de construção do conhecimento e não como resultante da falta de capacidade deles. Diante dessa realidade, a aplicação de provas desempenha outras funções, além das tradicionais, e a avaliação da aprendizagem passa a incluir a mediação do pensamento sintético, das aquisições e aplicações da aprendizagem, bem como a metacognição e as estratégias de aprendizagem dos estudantes, o que, de acordo com Sisto (2001), contribuiria para que o professor planeje o ensino de modo a potencializar a aquisição do conhecimento por parte de seus alunos, estabelecendo objetivos mais realistas e condizentes com as possibilidades deles e, como afirma Serrano (1991, p. 60), "contribuindo para a aprendizagem de atitudes mais positivas do aluno com relação ao seu desempenho".

Quanto às correlações entre o autoconceito e o desempenho acadêmico do grupo masculino e do feminino, não houve diferenças estatisticamente significativas. Tanto os universitários do gênero masculino quanto os do feminino apontaram com maior freqüência a categoria Autoconceito Bem Estruturado e com menor freqüência a categoria Autoconceito Supervalorizado. Os resultados obtidos são similares aos de outras pesquisas, como a de Castro (1999), que apontam que não há diferenças significativas ao se considerar 0 gênero dos sujeitos.

Também no que se refere à faixa etária, não foram constatadas diferenças significativas entre os grupos. Confirmam-se, assim, os resultados encontrados em estudos como o de Sossai (1975). No que diz respeito ao estabelecimento de correlações entre as duas variáveis focalizadas e o turno dos sujeitos analisados, a análise de variância revelou a existência de diferença significativa entre as médias semestrais e o autoconceito dos universitários do período diurno. Todavia não foram encontrados estudos que tenham realizado esse tipo de análise. Assumindo que o autoconceito é um constructo multidimensional e que todos os seres humanos são sensíveis aos estímulos ambientais e atribuem a eles um significado que resulta de suas crenças e opiniões, bem como das crenças e opiniões do ambiente em que estão inseridos, julgou-se importante investigar alguns aspectos relacionados ao autoconceito no que se refere ao contexto educacional e, especificamente, ao desempenho acadêmico de estudantes universitários. Assim, nesta pesquisa, foram enfocadas as percepções dos universitários a respeito de si próprios, recorrendo-se à Escala Reduzida do Autoconceito - ERA, e o desempenho acadêmico deles, aqui medido pelas médias semestrais obtidas nas disciplinas referentes ao primeiro semestre do ano letivo. 
Embora os objetivos do estudo tenham sido atingidos, é importante que se considerem as limitações metodológicas da presente pesquisa, especialmente no que se refere ao tamanho da amostra, ao instrumento utilizado e à sua circunscrição num único curso, de uma única instituição. Dessa forma, entende-se como necessário que outros trabalhos sejam realizados, considerandose inclusive o tipo de avaliação utilizada pelos docentes, possibilitando a exploração de outras amostras, instrumentos, cursos e áreas, garantindo a ampliação do conhecimento científico sobre 0 assunto.

\section{Referências}

Abu-Hilal, M. M. (2000). A Strutural model for predicting mathematics achievement: its relation with anxiety and self-concept in mathematics. Psychological Report, 86 (3/1), 835-847.

Albuquerque, C.M.S. \& Oliveira, C.P.F. (2002). Características psicológicas asso ciadas à saúde: a importância do autoconceito. Millenium - Revista do ISPV, 26.

Almeida, A. M. F. P. M. de (1997). Avaliação da aprendizagem e seus desdobramentos. Avaliação, $2(2 / 4), 37-50$.

Amorim, C. A. (2000). Modificação de autoconceito e rendimento escolar. Tuiuti: Ciência e Cultura, $20(20), 37-46$.

Brookver, W. B.; Thomas, S. \& Patterson, A. (1964). Self-concept of ability and school achievement. Sociology of Education, 37, 271-278.

Burns, R. B. (1979). The self-concept in theory, measurement and behaviour. London: Longman.

Bzuneck, J. A. (2001). O esforço nas aprendizagens escolares: mais do que um problema motivacional do aluno. Revista Educação e Ensino USF, 6(1),7-18.

Candieux, A. (1996). Psychometric properties of a pictorial self-concept scale among young learning disabled pupils. Psychology in the Schools, 33(3), 221-229.

Castro, N. I. M. de.(1999). Um estudo sobre a relação entre autoconceito, atribuição, meta- cognição e o desempenho acadêmico de alunos de segundo grau. Dissertação de Mestrado, Instituto de Psicologia, Universidade de São Paulo, São Paulo.

Corona, L. C. G. (2001). Escala Reduzida do Autoconceito - ERA. Rio de Janeiro: CEPA.

Cortesão, L., \& Torres, M.A. (1984). Avaliação pedagógica I: Insucesso Escolar. Porto: Porto .

Cubero, R. \& Moreno, M. C. (1995). Relações sociais nos anos escolares: família, escola, colegas. In: Coll, C., Palacios, J. \& Marchesi, A. (Orgs.). Desenvolvimento psicológico e educação: psicologia evolutiva. (pp. 190-202). Porto Alegre: Artes Médicas.

Engel, M. (1959). The stability os the self-concept in adolescence. The Journal of Abnormal and Social Psychology, (58), 211-215.

Estêvão, C. \& Almeida, L. S. (1999). Dimensões do autoconceito e sua relação com o rendimento escolar. Revista Psicologia Argumento, 17(24).

Hamachek, D. E.(1995). Self-concept and school achievement: interaction dynamics and a tool for assessing the self-concept component. Jounal of Counseling \& Development, (73), 419-425.

Housley, M. G.; Hooper, C. \& Gruber, M. B. (1998). Self-concept and motor performance of children with learning disabilities. Perceptual and Motor Skills, 87(3/1), 859-862.

Hugues, T. M. (1968). A study of the relationship of coping strength to self-concept, school achievement, and general anxiety level in sixth grade pupils. The Journal of Experimental Education, (37), 59-64.

Jacob, A.V. \& Loureiro, S.R. (1999). Autoconceito e o desempenho escolar. In: Programas e Resumos, 29. Reunião Anual de Psicologia. Campinas, 1999. Campinas: Sociedade Brasileira de Psicologia.

Jacob, A.V. (2001)O desempenho escolar e suas relações com autoconceito e auto-eficácia. Dissertação de Mestrado, Faculdade de Medicina, Universidade de São Paulo, Ribeirão Preto.

Jesus, D. M. De \& Gama, E. M. P. (1991). Desempenho escolar: sua influência no autoconceito e atitude em relação à escola. Cadernos 
de Pesquisa da UFES: Educação. Vitória: UFES/ PPGE, 1, 52-62.

Loureiro, S. R. (2001). O desempenho escolar, a motivação e as variáveis autoconceito e auto-eficácia. In: Resumos, 31. Reunião Anual de Psicologia. Rio de janeiro, 2001. Ribeirão Preto: Sociedade Brasileira de Psicologia.

Luckesi, C. C. (2001). Avaliação da aprendizagem escolar: estudos e proposições. (11 ed.) São Paulo: Cortez.

Marsh, H. W.(1984). Relations among dimensions of self-concept and academic achievements. Journal of Educational Psycology, (76),1291-1308.

Marsh, H.W., Byrne, B.M. \& Shavelson, R.J. (1988). A multifaceted academic self-concept: its relation to academic achievement. Journal of Educational Psycology, 80(3), 366-380.

Offer, D. (1971). The psychological world of the teen-ager: a study of normal adolescent boys. New York: Basic Books.

Pinto, A. L G. (1996). Avaliação formal e informal da aprendizagem em sala de aula. Psico-USF, 2(1), 13-38.

Serrano, M.R. (1991) O autoconceito e a percepção de controle e sua relação com o rendimento acadêmico. Dissertação de Mestrado, Faculdade de Educação, Universidade Estadual de Campinas, Campinas.

Sink, E. J.; Barnett, E. \& Pool, B. A. (1993). Perceptions of scholastic competence in relation to middle school achievement. Perceptual and Motor Skills, 76(2), 471-478.

Sisto, F. F. (2001). Rendimento acadêmico e processos cognitivos: algumas mudanças no paradigma da avaliação. In: Sisto, F. F., Sbardelini, E. T. B. \& Primi, R. (Orgs.), Contextos e questões da avaliação psicológica (pp. 117-134). São Paulo: Casa do Psicólogo.

Sordi, M. R. L. (2000). De Problematizando o papel da avaliação da aprendizagem nas metodologias inovadoras na área da saúde. Revista de Educação PUC-Campinas, (9), 52-61.
Sossai, J. A.(1975) Autoconceito: um estudo com adolescentes de São Paulo. Dissertação de Mestrado, Instituto de Psicologia, Universidade de São Paulo, São Paulo.

Sousa, S. M. Z. (1995). Avaliação da aprendizagem nas pesquisas no Brasil de 1930 a 1980. Caderno de Pesquisa, (94), 43-49.

Sousa, C. P. de; Depresbiteris, L; Franco, M. L. P. B. \& Sousa, S. Z. L (1995). Avaliação do rendimento escolar. (5 ed.) Campinas: Papirus.

Souza, E. M. de. (1996). Problemas de aprendizagem: crianças de 8 a 11 anos. Bauru: EDUSC.

Shavelson, R. J., \& Bolus, R. (1982). Self-concept: the interplay of theory and methods. Journal of Educational Psycology, 74(1), 3-17.

Stevanato, I. S. \& Loureiro, S. R. (2000). Autoconceito e aspectos comportamentais de crianças com dificuldades de aprendizagem. In: Jornada de Saúde Mental da Criança, 1., Ribeirão Preto, 2000. Ribeirão Preto: FMRP.

Vaz Serra, A. (1986). A importância do autoconceito. Psiquiatria Clínica, 7(2), 57-66.

Vaz Serra, A. (1988). O autoconceito. Análise Psicológica, 2(6), 101-110.

Vaz Serra, A. \& Firmino, H. (1986). O autoconceito nos doentes com perturbações emocionais. Psiquiatria Clínica, 7(2), 91-96.

Veiga, F.H. (1988). Disciplina materna, autoconceito e rendimento escolar. Cadernos de consulta psicológica: família e desenvolvimento humano, 4, 47-56.

Veiga, F.H. (1989). Escala de autoconceito: Adaptação portuguesa do "Piers-Harris Children's SelfConcept Scale”. Psicologia, 3(7),275-284.

Wylie, R. C. (1961). The self-concept: a critical survey of pertinent research literature. Lincoln: University of Nebraska Press.

Recebido em/received in: 4/09/2005 Aprovado em/approved in: 13/11/2005 